\title{
THE DIRICHLET PROBLEM FOR A COMPLEX MONGE-AMPERE EQUATION
}

\author{
BY ERIC BEDFORD 1 AND B. A. TAYLOR 2
}

Communicated by P. R. Halmos, September 25, 1975

On $\mathbf{C}^{n}$, write $d=\partial+\bar{\partial}, d^{c}=i(\bar{\partial}-\partial)$ so that $d d^{c} u=2 i \partial \bar{\partial} u$, and let

$$
\beta_{n}=\left(\frac{i}{2}\right)^{n} \prod_{j=1}^{n} d z_{j} \wedge d \bar{z}_{j}
$$

be the usual volume form. We study here the nonlinear Dirichlet problem,

$$
\left(d d^{c} u\right)^{n}=d d^{c} u \wedge \cdots \wedge d d^{c} u=f \beta_{n} \quad \text { on } \Omega,
$$

$$
\begin{gathered}
u \text { plurisubharmonic on } \Omega, \\
u=\phi \text { on } \partial \Omega
\end{gathered}
$$

where $\Omega$ is a bounded open set in $\mathbf{C}^{n}, f \geqslant 0$, and $\phi$ is a continuous function on $\partial \Omega$. For arbitrary plurisubharmonic functions $u$, it is known that $d d^{c} u$ is a positive current of type $(1,1)[4$, p. 70$]$; but, it is not clear that the higher exterior powers of $d d^{c} u$ are well defined. In fact, examples indicate that it is probably not possible to define $\left(d d^{c} u\right)^{n}$ as a distribution for all plurisubharmonic functions $u$ [7]. However, for bounded, $C^{2}$ plurisubharmonic functions, Chern, Levine, and Nirenberg [3] have given an estimate which makes it clear how to define $\left(d d^{c} u\right)^{n}$ when $u$ is a continuous plurisubharmonic function. If $\|u\|_{\Omega}=$ $\sup \{|u(z)|: z \in \Omega\}$, then they prove that for each compact subset $K$ of $\Omega$, there is a constant $C=C(K)$ such that

$$
\int_{K}\left(d d^{c} u\right)^{n} \leqslant C\left\{\|u\|_{\Omega}\right\}^{n}
$$

for all $C^{2}$ plurisubharmonic functions $u$ on $\Omega$. With this result (and its proof), it is easy to show that the operator $\left(d d^{c} u\right)^{n}$, thought of as a mapping from the $C^{2}$ plurisubharmonic functions on $\Omega$ to the space of nonnegative Borel measures on $\Omega$, has a continuous extension to the space of all continuous plurisubharmonic functions on $\Omega$. It is with this definition of $\left(d d^{c} u\right)^{n}$ as a nonnegative Borel measure on $\Omega$ that we study the Dirichlet problem (1).

AMS (MOS) subject classifications (1970). Primary 32 F05, 35D05; Secondary 32 E99.

${ }^{1}$ Research supported in part by a Sloan Foundation Grant to Courant Institute of Mathematical Sciences, New York University, and by the Army Research Office grant number DAHC04-75-G-0149.

${ }^{2}$ Research supported in part by the National Science Foundation Grant Number GP 37628 . 
THEOREM 1. Let $\Omega$ be a bounded, strongly pseudoconvex set in $\mathbf{C}^{n}$ with $C^{2}$ boundary. Let $\phi \in C(\partial \Omega)$ and $f \geqslant 0, f \in C(\bar{\Omega})$. Then there exists a unique solution to the Dirichlet problem (1) in the class of all functions continuous on $\bar{\Omega}$ and plurisubharmonic in $\Omega$.

The differential equation of (1) bears a strong resemblance to the real Monge-Ampere equation (see, e.g. [1] , [5] , [6]). However, in contrast to the situation for real Monge-Ampere equations there are, in general, many different (but not continuous) plurisubharmonic functions solving (1), at least when the nonnegative measure $\mu=f \beta_{n}$ of (1) is allowed to be singular. Thus, uniqueness is not possible without some mild regularity assumption.

Our method of proof is the familiar Perron method. The solution is exhibited as the upper envelope of a family of subsolutions. The uniqueness part follows from the following minimum principle.

THEOREM 2. Let $\Omega$ be a bounded open set in $\mathbf{C}^{n}$. If $u, v$ are continuous on $\bar{\Omega}$, plurisubharmonic in $\Omega$, and if $\left(d d^{c} u\right)^{n} \leqslant\left(d d^{c} v\right)^{n}$ in $\Omega$, then

$$
\min \{u(z)-v(z): z \in \Omega\}=\min \{u(z)-v(z): \mid z \in \partial \Omega\}
$$

In a special case, we also prove a regularity result.

THEOREM 3. If $\Omega$ is the unit ball in $\mathrm{C}^{n}$, if $\phi \in C^{2}(\partial \Omega)$, and if $f^{1 / n} \in$ $C^{2}(\bar{\Omega})$, then the unique, continuous plurisubharmonic solution of (1) has locally bounded second partial derivatives.

In general, the solution $u$ of problem (1) will not be of class $C^{2}$ when $f$ $\equiv 0$, even if $\phi$ is real analytic.

Under stronger conditions, the Laplacian of $u$ also satisfies a maximum principle.

THEOREM 4. If $u \in C^{2}(\bar{\Omega}), u$ is plurisubharmonic on $\Omega$, and $u$ solves (1), with $f \in C(\bar{\Omega})$ a nonnegative, plurisubharmonic function on $\Omega$, then for $1 \leqslant j$ $\leqslant n$,

$$
\max \left\{\frac{\partial^{2} u}{\partial z_{j} \partial \bar{z}_{j}}(z): z \in \Omega\right\}=\max \left\{\frac{\partial^{2} u}{\partial z_{j} \partial \bar{z}_{j}}(z): z \in \partial \Omega\right\}
$$

One interest in the Dirichlet problem (1) results from the work of Bremermann [2], who introduced the following function. Given $\phi \in C(\partial \Omega)$, let $B(\phi, \Omega)$ denote the Perron-Bremermann family of all plurisubharmonic functions $v$ on $\Omega$ such that $\lim \sup _{z \rightarrow \zeta} v(z) \leqslant \phi(\zeta)$ for all $\zeta \in \partial \Omega$, and then define

$$
(S \phi)(z)=\sup \{v(z) \mid v \in B(\phi, \Omega)\}
$$


$\rightarrow \zeta \in \partial \Omega$, provided that $\Omega$ is strongly pseudoconvex. It was later proved by J. B. Walsh [8] that $S \phi$ is continuous on $\bar{\Omega}$. Bremermann also noted that if $S \phi \in C^{2}(\Omega)$, then $\left(d d^{c} u\right)^{n}=0$ in $\Omega$. Now, in general, $S \phi$ is not a $C^{2}$ function, but in any case, Theorem 1 shows that $S \phi$ is characterized as the unique continuous solution of the special case of (1) with $f \equiv 0$.

Bremermann also proved that the envelope of holomorphy of the Hartogs' domain in $\mathbf{C}^{n+1},\left\{(z, w): z \in \partial \Omega,|w| \leqslant \exp (-\phi(z))\right.$, or $\left.z \in \Omega,|w| \leqslant e^{-M}\right\}, M$ $=\max \{\phi(z): z \in \partial \Omega\}$, is described as $\left\{(z, w) \in \mathrm{C}^{n+1}:|w| \leqslant \exp (-S \phi(z)), z\right.$ $\in \bar{\Omega}$ \}, when $\Omega$ is strictly pseudoconvex. Thus, the boundary of this envelope of holomorphy is characterized as the solution to the partial differential equation $\left(d d^{c} u\right)^{n}=0$. In particular, if $\Omega$ is the unit ball in $\mathbf{C}^{n}$ and $\phi$ is smooth, then Theorem 3 yields a smoothness property of the boundary of this envelope of holomorphy.

\section{BIBLIOGRAPHY}

1. A. D. Alexandrov, Dirichlet's problem for the equation Det $\left\|z_{i, j}\right\|=$ $\phi\left(z_{1}, \ldots, z_{n}, x_{1}, \ldots, x_{n}\right)$. I., Vestnik Leningrad Univ. Ser. Mat. Meh. Astr. 13 (1958), no. 1, 5-24. (Russian) MR 20 \#3385.

2. H. J. Bremermann, On a generalized Dirichlet problem for plurisubharmonic functions and pseudo-convex domains. Characterization of Šlov boundaries, Trans. Amer. Math. Soc. 91 (1959), 246-276. MR 25 \#227.

3. S. S. Chern, Harold I. Levine and L. Nirenberg, Intrinsic norms on a complex manifold, Global Analysis (Papers in honor of K. Kodaira), Univ. of Tokyo Press, Tokyo, 1969, pp. 119-139.

4. P. Lelong, Fonctions plurisousharmoniques et formed différentielles positives, Gordon and Breach, New York, 1968. MR 39 \#4436.

5. A. V. Pogorelov, Monge-Ampere equations of elliptic type, Izdat. Har'kovsk. Univ., Kharkov, 1960; English transl., Noordhoff, Groningen, $1964 . \quad$ MR 23 \#A1137; 31 \#4993.

6. The Dirichlet problem for the n-dimensional analogue of the MongeAmpere equation, Dokl. Akad. Nauk SSSR 201 (1971), 790-793 = Soviet Math. Dokl. 12 (1971), 1727-1731. MR 45 \#2305.

7. Y. T. Siu, Extension of meromorphic maps, Ann. of Math. (to appear).

8. J. B. Walsh, Continuity of envelopes of plurisubharmonic functions, J. Math. Mech. 18 (1968/69), 143-148. MR 37 \#3049.

COURANT INSTITUTE OF MATHEMATICAL SCIENCES, NEW YORK UNIVERSITY, NEW YORK, NEW YORK 10012

DEPARTMENT OF MATHEMATICS, UNIVERSITY OF MICHIGAN, ANN ARBOR, MICHIGAN 48104 